\title{
Targeting Gastric Cancer with Trastuzumab: New Clinical Practice and Innovative Developments to Overcome Resistance
}

\author{
Dimitrios H. Roukos, MD \\ Department of Surgery, Personalized Cancer Medicine, Biobank, Ioannina University, Ioannina, Greece
}

At last, following decades of research endeavor, clinical success is here. A new molecular classification and novel targeted therapy can improve poor oncological outcomes of some patients with gastric cancer.

Each year 900,000 patients are diagnosed worldwide with gastric cancer. Potentially, approximately $25 \%$ of these patients may benefit by adding trastuzumab to current standard treatment. Why and what is the new molecular classification crucial for this therapeutic decision? What are the limitations of this monoclonal antibody, and how could intrinsic and acquired resistance be overcome?

Despite optimization of surgery, radiotherapy, and cytotoxic chemotherapy, survival of advanced gastric cancer is poor. Five years after this multimodal treatment, $<40 \%$ of Western patients with stage II or III disease are alive. In metastatic stage IV, mean survival is only 10 months.

Most promise to improve this poor survival is provided by biologically targeted agents. The concept is exciting. Suppression of deregulated signaling pathways which play a central role in cell proliferation, survival, apoptosis, and angiogenesis may be a highly effective approach against cancer. Over the last decade, several agents targeting key components of important downstream signaling have been developed and approved by the Food and Drug Administration (FDA) for a series of cancers. Inhibition of signaling cascades may suppress cancer cell proliferation and survival. However, for most solid tumors, clinical efficacy measured by overall survival benefit is modest. Despite initial enthusiasm based on preclinical and phase II clinical studies, subsequent phase III randomized controlled trials were negative. However, some other randomized trials have demonstrated clinical utility in selected patients. ${ }^{1}$

(C) Society of Surgical Oncology 2009

Published Online: 20 October 2009

D. H. Roukos, MD

e-mail: droukos@cc.uoi.gr

\section{FROM BASIC SCIENCE DISCOVERY TO CLINICAL PRACTICE}

The discovery of the epidermal growth factor (EGF) and its receptor (EGFR) in 1962 and 1978, respectively, opened the way for a new era of molecular oncology. ${ }^{2}$ However, successful translation of these basic research findings into the clinic has occurred only during the last decade and mostly for only one type of cancer, i.e., breast cancer. ${ }^{1}$

The Erbb family consists of four closely related type 1 transmembrane tyrosine kinase receptors: EGFR (or HER1), ERBB2 (HER2), ERBB3 (HER3), and ERBB4 (HER4). Each receptor comprises an extracellular domain at which ligand binding occurs, an $\alpha$-helical transmembrane segment, and an intracellular protein tyrosine kinase domain. Ligand binding to these EGF family receptors phosphorylates and activates a complex intracellular signaling pathways network that controls a range of cellular processes including proliferation, angiogenesis, cell cycle, survival, and apoptosis (Fig. 1a). ${ }^{3}$

HER2 amplification and overexpression plays a central role in initiation, progression, and metastasis of some common cancers, including breast cancer and gastric cancer. ${ }^{1-4}$ HER2 status has been recognized as an important prognostic factor. Patients with breast cancer or gastric cancer and HER2-positive disease have significantly worse survival than those with HER2-negative tumors. ${ }^{3,4}$ Thus, this pivotal receptor is a potential therapeutic target.

Trastuzumab binding (Fig. 1b) inhibits HER2 signaling pathway activity in tumor cells overexpressing HER2. Phase III trials confirming preclinical and clinical data for the safety and efficacy of trastuzumab independently of robust clinicopathologic factors in both metastatic and adjuvant setting have led to the establishment of this antibody as standard treatment for HER2-positive breast cancer. $^{1-3}$ However, there has been no such evidence for any other cancer. 
a

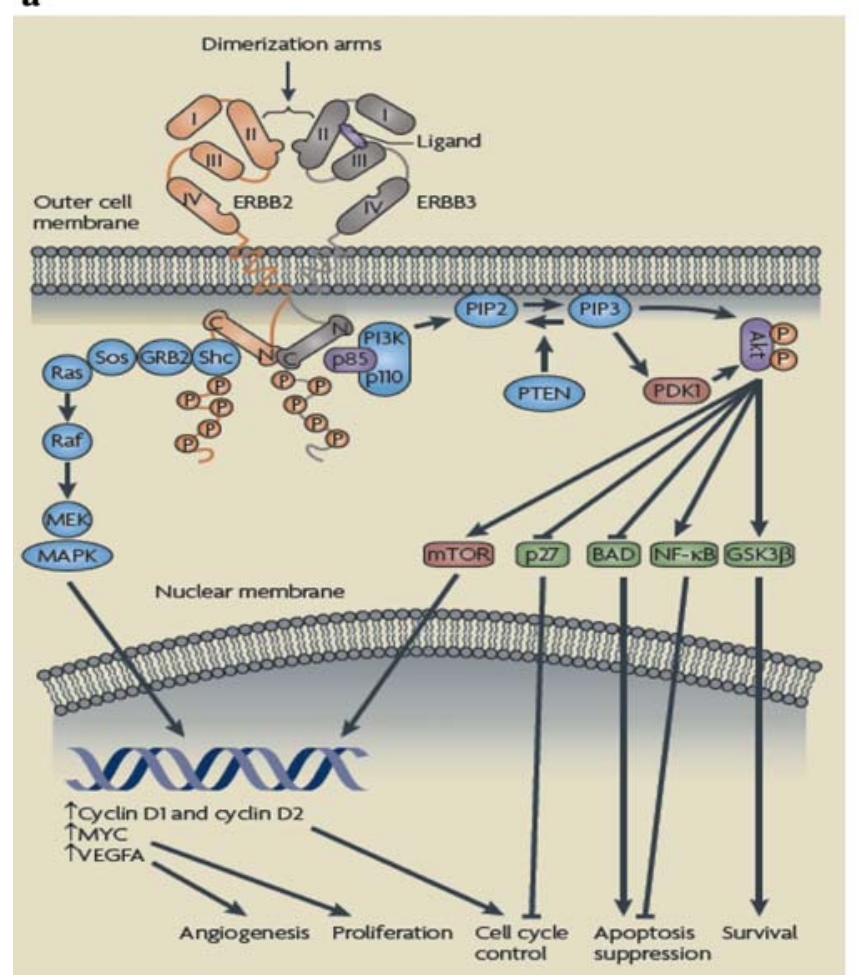

b

I Inhibition through direct
antibody binding

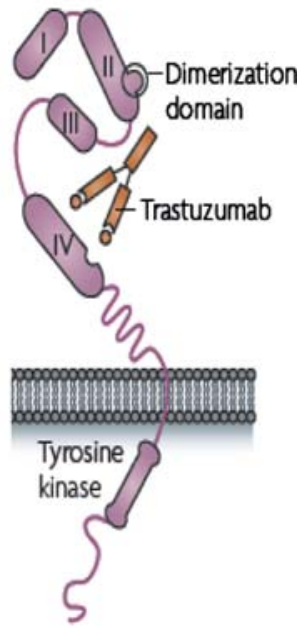

FIG. 1 a Ligand binding and subsequent Erbb dimer formation initiates signaling through a complex array of intracellular pathways that initiate and control a range of cellular processes. Dimer formation results in the cross-phosphorylation of the dimer partners, creating docking sites that allow the recruitment of downstream signaling components and the formation of signaling complexes. Two key signaling pathways activated by the Erbb family dimers are the MAPK pathway, which stimulates proliferation, and the PI3K-Akt pathway, which promotes tumor cell survival (see the figure). Only signaling through these two pathways and some of the known

\section{CHANGING TREATMENT OF GASTRIC CANCER}

Now, for the first time, positive results of a phase III trial for the efficacy of trastuzumab are reported for gastric cancer. Van Cutsem and colleagues have presented the results of the ToGA study in the 2009 ASCO Annual Meeting, May 29-June 2, in Orlando, FL. ${ }^{5}$ In this randomized controlled multicenter trial, 594 patients were randomized 1:1 at sites in Europe, Latin America, and Asia. All these patients had HER2-positive gastroesophageal and gastric adenocarcinoma (locally advanced, recurrent or metastatic). They were randomized to receive trastuzumab (Herceptin) and chemotherapy (5-fluorouracil or capecitabine and cisplatin) for six cycles or chemotherapy alone. Trastuzumab was given until disease progression.

Addition of trastuzumab to chemotherapy improved oncological outcomes. Median overall survival was significantly longer (13.5 months) in the experimental arm (trastuzumab plus chemotherapy) than in the standard arm outcomes are shown here for simplicity. b The antibody trastuzumab binds directly to domain IV of the extracellular region of ERBB2, suppressing ERBB2 signaling activity, preventing cleavage of the extracellular domain, and marking tumor cells that overexpress ERBB2 for further immunological attack through antibody-dependent cell-mediated cytotoxicity. GSK3 $\beta$, glycogen synthase kinase $3 \beta$; NF$\kappa \mathrm{B}$, nuclear factor- $\kappa \mathrm{B}$; PDK1, pyruvate dehydrogenase kinase 1 ; PIP2, phosphatidylinositol biphosphate; PIP3, phosphatidylinositol triphosphate

(chemotherapy alone; 11.1 months) $[P=0.0048$; hazard ratio (HR) 0.74 ; 95\% confidence interval (CI) $0.60-0.91]$. Overall response rate was significantly increased by $13 \%$ in the trastuzumab arm $(P=0.0017)$. Safety profile and adverse effects data showed that trastuzumab-based regimen was a well-tolerated treatment; there was no difference in symptomatic congestive heart failure between arms, and asymptomatic left ventricular ejection fraction decreases were reported as $4.6 \%$ in the experimental arm and $1.1 \%$ in the chemotherapy arm.

This study provides a series of strengthens and a few limitations. Based on evidence available from previous positive and negative clinical trials with targeted agents, the investigators have appropriately designed this study. First, only HER2-positive patients were included. Tumors from 3,807 patients were centrally tested for HER2 status: this centralization ensures reliable assessment, quality control, and accurate response and survival rates given that only HER 2 patients were recruited. The rate of $22 \%$ for 
HER2-positive gastric cancer is similar to the HER2positive breast cancer rate. Second, the investigators correctly decided to use overall survival as primary endpoint and not progression-free survival (PFS). Indeed, the objectivity of PFS to assess response, efficacy, and clinical utility of an experimental targeted agent has become questionable. Cancer heterogeneity is one of the major biological arguments against the use of PFS to measure therapy efficacy. Although several targeted agents have been approved by the FDA based on significant improvement of PFS, more current evidence suggests that some cancer cell populations, initially rare within the tumor, refuse to die under treatment. ${ }^{6}$ Therefore, a nonprogressive disease assessment by imaging techniques (no tumor size increase) does not reflect overall response. Sensitive cancer cells are killed, but resistant cells proliferate, developing a uniform tumor consisting of resistant cells. These cancer cells have the ability of metastasis, which results in no overall survival benefit.

\section{LIMITATIONS}

The absolute benefit in response rate to trastuzumab addition to chemotherapy was $12.8 \%$ and it resulted in a prolongation of overall survival by 2.4 months. These data indicate resistance to trastuzumab even among HER2positive selected patients. However, it is likely that, because of less residual disease after surgery in resectable gastric cancer, the trastuzumab therapeutic gain may be larger in the adjuvant setting with substantial 5-year survival rate benefit. However, we should await the results of such new adjuvant phase III trastuzumab trials.

\section{CLINICAL PRACTICE}

Although we should await details of the ToGA study fulltext publication, it appears that a new classification-based treatment decision is now being considered. HER2 status assessment appears essential in the diagnostic workup of patients with locally advanced, recurrent or metastatic adenocarcinoma of esophagogastric junction and stomach. Although a similar trastuzumab effect is likely in the adjuvant setting, considering also the HER2-positive breast cancer experience, no trastuzumab use for early HER2positive gastric cancer can be suggested before the completion of new trastuzumab-based phase III adjuvant trials.

\section{PERSPECTIVES FOR OVERCOMING RESISTANCE}

Resistance to molecular targeting therapy is currently the cause of treatment failure in cancer. Despite trastuzumab-containing treatment a substantial proportion of HER2-positive breast cancer patients either recur in the adjuvant setting or progress after initial response and die of the disease. Similarly, the absolute additional response rate to trastuzumab among HER2-positive advanced gastric cancer in the ToGA study is small: $12.8 \%$. Given that HER2-positive accounts for approximately 25\%, only $3.12 \%$ of all gastric cancer patients can benefit from trastuzumab treatment.

How could this intrinsic or acquired resistance be overcome? Research strategies are focused on the development of both novel drugs and molecular markers beyond HER2 expression for tailoring the best treatment to individual patients. There are two main directions: first, better understand of Erbb signaling pathways and trastuzumab mechanisms of actions and resistance; second, exploring the role of other signaling pathways including $\mathrm{Wnt} / \mathrm{b}$ catenin, TGF-b/SMADs, and other pathways involved in cancer may lead to understanding of intracellular signaling pathways network in various cancer types.

The first, more realistic, approach has already led to clinical applications. Improved insights into the biology of the Erbb family have led to additional active anti-HER2 therapies. New strategies against HER2 include Erbb tyrosine kinase inhibitors (TKIs), heat shock protein 90 inhibitors, Erbb dimerization inhibitors, and antibodychemotherapy conjugates. All of these approaches have shown substantial clinical activity in patients who have progressed on trastuzumab treatment. ${ }^{3}$ TKIs-based targeting of HER2, preventing signal transduction of both the Ras-RAF1 MAPK and PI3K-Akt pathways, led to an increase in apoptosis and a decrease in cellular proliferation (Fig. 1).

The most clinically advanced anti-HER2 TKI is lapatinib (Tykerb; GlaxoSmithKline), a dual HER2 and EGFR TKI small molecule. Based on a pivotal phase III study, lapatinib in combination with capecitabine has been approved by the FDA for use in patients with advanced HER2-positive breast cancer who have previously received trastuzumab. ${ }^{3}$ Therefore, lapatinib alone or in combination with trastuzumab, which was shown to be superior to a single agent, are potential effective therapies to overcome trastuzumab resistance in HER2-positive gastric cancer. ${ }^{3}$

Mutations in signaling components downstream of the initiating Erbb dimer and several interconnections between the two main signaling pathways MAPK and PI3 K-Akt of Erbb may bypass therapy suppression and are plausible explanations for resistance to trastuzumab in HER2-positve breast cancer and possibly also gastric cancer. Multitargeted approaches have been evaluated and provided promising results; for example, encouraging findings have been reported with inhibition of mToR activity or the addition of a dual inhibitor of both mToR and 
PI3K (Fig. 1) to overcome resistance to lapatinib and trastuzumab. ${ }^{3}$

\section{MULTITARGETING, SIGNALING PATHWAYS NETWORK-BASED THERAPY}

Although still in its infancy, the second approach to predict complex signaling pathways interactions, including Erbb signaling, if successful, might revolutionize treatment of gastric cancer, breast cancer, and other solid tumors. Given the current strong evidence that multiple genetic alterations and several signaling pathways are dysregulated in solid cancers, one of the most rational approaches is to inhibit these pathways. ${ }^{7}$ Combining targeted agents and considering crosstalk between pathways and bypass of targeted agents as well as predictors of response might lead to highly effective therapies. ${ }^{7}$

However, there are many challenges. Cancer heterogeneity is reflected by variation in deregulated pathways among patients with the same tumor, tumor-node-metastasis (TNM) staging, and clinicopathologic factors. At present there is no standard method to identify either which pathways are dysregulated or how they interact in individual patients. The new era of personalized medicine provides major promises. One approach is to integrate personal genomics and clinicopathologic and treatment data into sophisticated in silico models to predict genotype-phenotype map in cancer. ${ }^{8}$ Rapid advances in molecular systems biology and future cheaper whole-genome cancer data scans are innovative exciting developments towards the development of novel response predictors and a new generation of multitargeted agents. ${ }^{8,9}$ The new era of personalized cancer care is here, but multiple challenges including major funding requirements and reliable data analysis make the translation of personalized research approaches into clinical medical practice difficult. ${ }^{10}$

\section{CONCLUSIONS}

HER2 status should now be included in diagnostic makeup of patients with advanced gastric cancer. Addition of trastuzumab to chemotherapy improves overall survival and is a new standard treatment for patients with locally advanced, recurrent or metastatic HER2-positive disease. Although this efficacy is likely in the adjuvant setting, an evidence-based decision on trastuzumab use in early gastric cancer requires the completion of new adjuvant phase III trials.

Resistance to current therapies is a major challenge. Lapatinib and other novel antibodies or TKIs tested in clinical trials for HER2-positive breast cancer might also prove effective in trastuzumab-resistant HER2-positive gastric cancer. However, such Erbb-based approaches have less application in HER2-negative disease, which accounts for the majority of patients with gastric cancer or breast cancer. Understanding genotypic-phenotypic cancer diversity and signaling feedback loops as well as developing reliable methods to screen for identifying dysregulated signaling pathways in individual patients is a rational and exciting approach. If successful, such comprehensive approaches using molecular systems biology and future whole-genome cancer data scans may result in the discovery of novel multitargeted therapies tailored to individual patients on the basis of novel predictors of response to combined therapies.

ACKNOWLEDGMENT There is no research support for this article.

\section{REFERENCES}

1. Burstein HJ. The distinctive nature of HER2-positive breast cancers. N Engl J Med. 2005;353(16):1652-4.

2. Gschwind A, Fischer OM, Ullrich A. The discovery of receptor tyrosine kinases: targets for cancer therapy. Nat Rev Cancer. 2004;4(5):361-70.

3. Baselga J, Swain SM. Novel anticancer targets: revisiting ERBB2 and discovering ERBB3. Nat Rev Cancer. 2009;9(7):463-75.

4. Gravalos C, Jimeno A. HER2 in gastric cancer: a new prognostic factor and a novel therapeutic target. Ann Oncol. 2008;19(9): $1523-9$

5. Van Cutsem E, Kang Y, Chung H, et al. Efficacy results from the ToGA trial: A phase III study of trastuzumab added to standard chemotherapy (CT) in first-line human epidermal growth factor receptor 2 (HER2)-positive advanced gastric cancer (GC). J Clin Oncol. 2009;27:18s (suppl; abstr LBA4509).

6. Bastiaens P. Systems biology: when it is time to die. Nature. 2009;459(7245):334-5.

7. Jones S, Zhang X, Parsons DW. Core signaling pathways in human pancreatic cancers revealed by global genomic analyses. Science. 2008;321(5897):1801-6.

8. Roukos DH. Personalized cancer diagnostics and therapeutics. Expert Rev Mol Diagn. 2009;9(3):227-9.

9. Diestock TS. Personalizing medicine: a systems biology perspective. Mol Syst Biol. 2009;5:249. Epub 2009 Mar 17.

10. Roukos DH. Mea Culpa with cancer-targeted therapy: new thinking and new agents design for novel, causal networks-based, personalized biomedicine. Expert Rev Mol Diagn. 2009;9(3): $217-21$. 